\title{
Application of dry-ice for transient spray cooling
}

\author{
Miguel R. Oliveira Panão*, José J. Costa, Mário Bernardo \\ ADAI-LAETA, Universidade de Coimbra, Portugal \\ ${ }^{*}$ Corresponding author: miguel.panao@dem.uc.pt
}

\begin{abstract}
Spray cooling systems are able to remove large amounts of heat due to phase-change. Although vaporization is the most common phase-change process used in applications requiring thermal management, the use of liquids often implies the presence of a liquid film which is known to mitigate cooling performance. Thus, it is worth exploring other approaches for spray cooling avoiding liquid films. The work presented here explores sublimation using $\mathrm{CO}_{2}$ particles (dry-ice) formed through the Joule-Thomson effect. The application of interest is the molding industry, where reducing the cycle time taking advantage of the time-frame available between the mold opening and closing during the part's extraction, allows a production increase and, consequently, a higher competitive advantage in the market. The purpose of the experiments performed in dry-ice particle spray cooling is to investigate the effect of the impingement distance $(350-450 \mathrm{~mm})$, and injection duration, on the total energy flux removed from the surface, and cooling efficiency, in order to assess the performance of sublimation spray cooling. The results show an evolution of temperature distribution from a more homogeneous pattern with shorter pulses to a heterogeneous one for pulse durations longer than $1 \mathrm{~s}$. This is particularly useful in hotspot cooling. In terms of changing the spray impact distance, the higher particle dispersion achieved with a larger distance led to a decrease in thermal performance, probably due to the saturation of $\mathrm{CO}_{2}$ close to the impact surface. However, the pattern observed for the evolution of the total energy flux removed, with a maximum around an injection duration of $0.5 \mathrm{~s}$, remains unaltered. The maximum cooling efficiency, obtained for the shortest distance, is up to $30 \%$, which is comparable to spray cooling systems based on vaporization.
\end{abstract}

\section{Keywords}

Dry-Ice, spray cooling, molding processes

\section{Introduction}

The optimization of cycle times in molding processes depends on the ability to improve the cooling period. Wire coil inserts inside water channels already contribute to enhance heat transfer. However, the time between the extraction of the plastic molded part and closing for initiating the next cycle presents an opportunity for an additional cooling. Although most phase-change technique involving sprays consider liquid coolants, our purpose is to avoid the formation of liquid films. Therefore, this work explores sublimation phase-change using a dry-ice (solid $\left.\mathrm{CO}_{2}\right)$ particle spray to assess its potential for reducing cycle times in molding processes.

There are few cooling systems using dry-ice particles. To the best of our knowledge, only Linde developed a patented spot cooling system using $\mathrm{CO}_{2}$ to perform thermal management in local hotspots of molded parts [1]. However, the process operates in steady-state and may not apply in short transient cooling.

Most applications using dry-ice sprays aim at surface cleaning due to the solvent properties of $\mathrm{CO}_{2}$ particles [2]. Only a few studies consider the thermal effects of its impact on a surface, and even less consider their application to develop thermal management systems. For low flow rates $(0.236-1.18 \mathrm{l} / \mathrm{s})$, Kim and Lee [3] characterized the heat transfer coefficient $(h)$ in steady-state conditions, measuring values between 1 and $3.5 \mathrm{~kW} \cdot \mathrm{m}^{-2} \mathrm{~K}^{-1}$. For the highest value of $h$, the stagnation temperature varied between $0^{\circ} \mathrm{C}$ and $16^{\circ} \mathrm{C}$ depending on the impingement distance. In fact, increasing the distance from 5 to 20 times with a diameter at nozzle exit of $d=1 \mathrm{~mm}$ led to a non-linear decrease of $h$ by $40 \%$. The authors conclude that dry-ice particles are an advantage in $\mathrm{CO}_{2}$ jet cooling, making it a better suited system for cooling applications. However, there are applications where $\mathrm{CO}_{2}$ particle sprays have a short time frame to cool a heated surface, meaning it is operating in highly transient conditions. In this sense, the analysis of the corresponding transient thermal behaviour is still lacking in the literature and, thus, is the motivation for this work.

Using a mass flow rate of the order of $\sim 10^{2} \mathrm{~g} / \mathrm{s}$, the present investigation focuses on the transient heat transfer in sublimation spray cooling for several distances between the diffuser exit and the impinging heated surface. The experiments devised allow quantifying the cooling potential of dry ice particle sprays, assessing its potential application in the transient thermal management of molds. The purpose is to explore the short time of mold opening for the part's extraction before starting another injection cycle by achieving a significant heat removal from the molding cavity surface.

\section{Experimental Setup}

The experimental setup comprises a $5 \mathrm{~kg} \mathrm{CO}_{2}$ fire extinguisher vessel with two laser pointers assembled in the diffusor to align it with the center of the impinging surface. The pressure inside the vessel is at $57.3 \mathrm{bar}$ at $20^{\circ} \mathrm{C}$ and an inner probe-tube submerged down to the vessel bottom ensures that liquid $\mathrm{CO}_{2}$ arrives at the nozzle exit. 
In each experiment, the weight difference of the reservoir between the start and the end of injection, divided by its duration, provides the average mass flow rate, resulting in a value of $\dot{m}=154 \pm 2.95 \mathrm{~g} / \mathrm{s}$. The rapid expansion of the liquid $\mathrm{CO}_{2}$ through the nozzle and diffuser implies a sudden temperature decrease towards the sublimation value at atmospheric pressure, forming a two-phase (solid-gas) mixture by the Joule-Thomson effect (process a-b in Fig. 1).

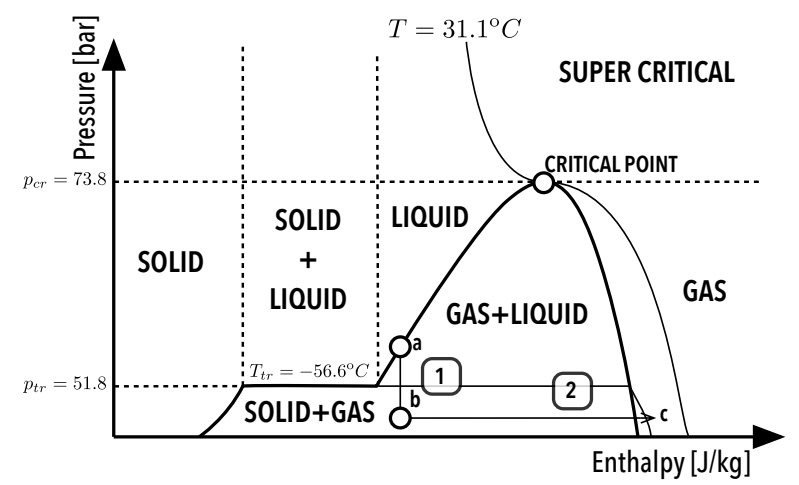

Figure 1. $\mathrm{CO}_{2}$ phase diagram. Process 1 corresponds to the expansion from points a to $\mathrm{b}$ producing a two-phase mixture of gas and $\mathrm{CO}_{2}$ solid particles. Process 2 corresponds to the sublimation associated with surface cooling.

The plate made of AISI 1010 steel has dimensions of $290 \times 290 \times 5[\mathrm{~mm}]$ and its thermophysical properties at $45^{\circ} \mathrm{C}$ (based on the average value measured throughout the experiments) are: thermal conductivity of $k=$ $63 \mathrm{~W} \cdot \mathrm{m}^{-1} \mathrm{~K}^{-1}$, specific heat $c_{p}=443.5 \mathrm{~J} \cdot \mathrm{kg}^{-1} \mathrm{~K}^{-1}$, density $\rho=7832 \mathrm{~kg} / \mathrm{m}^{3}$. Before each test, the plate is preheated with a $200 \mathrm{~W}$ electric resistance up to $100-110^{\circ} \mathrm{C}$, and then placed on a horizontal cork, $40 \mathrm{~mm}$-thick insulation board, its center aligned with the vertical diffuser axis. This configuration ensures a uni-direction internal conduction heat flux inside the steel plate, normal to the impinging surface. The experiments performed consider an initial condition of uniform surface temperature of $90^{\circ} \mathrm{C}$, since this is a common value found in molds upon releasing the molded part. Fig. 2 depicts an overall view of the experimental setup and main flow structures, including the spray dispersion pattern visualized through its angle, the impact region and a toroidal vortex formed by the micro- $\mathrm{CO}_{2}$-particles following the gas flow.
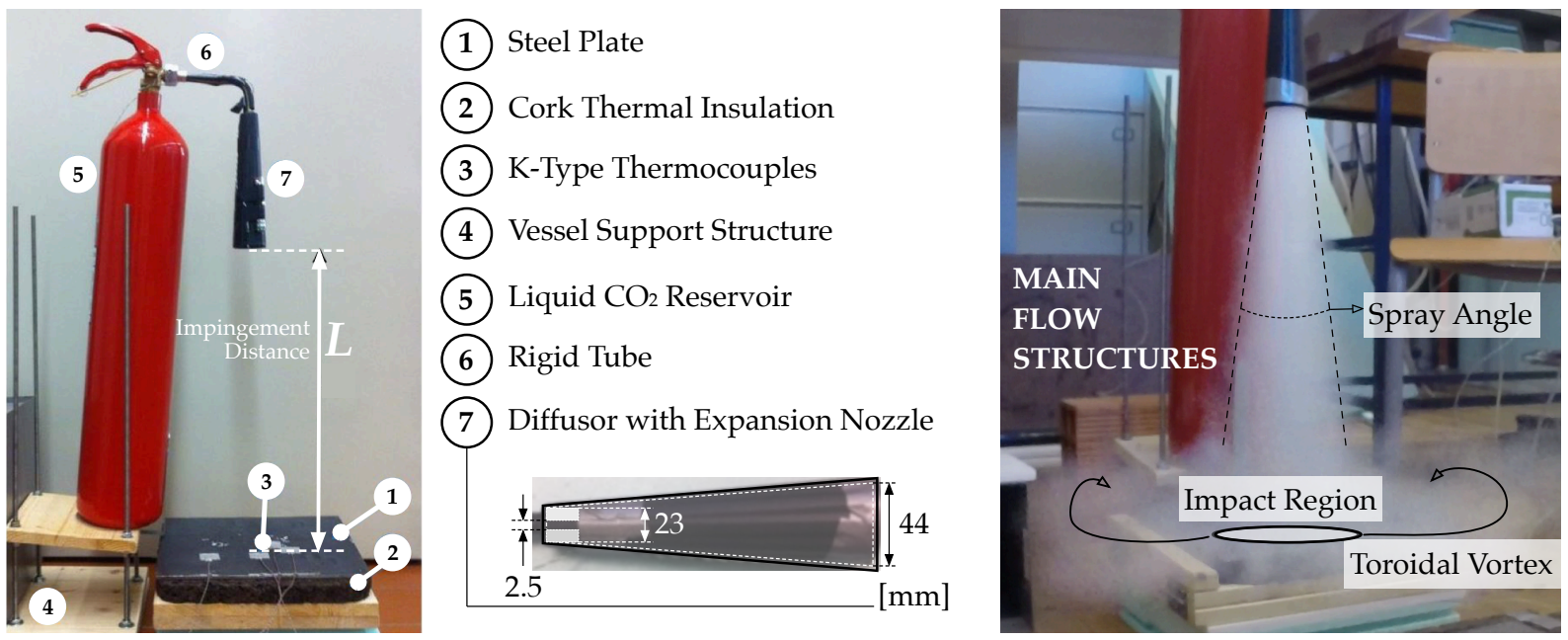

Figure 2. Overall view of experimental setup and indication of main flow characteristics.

The temperature measured by K-type Omega cement-on thermocouples allows characterizing the surface thermal behavior. A thin, highly polished aluminium tape fixes each thermocouple to the impinging surface, with a negligible thermal resistance, and minimizing radiation heat losses. A data logger from Data Translation (DT9828) acquires temperature data at a sampling rate of $120 \mathrm{~Hz}$ in each thermocouple channel. Calculations of the local heat flux from surface temperature measurements allow characterizing the cooling effect at the dry ice particles spray impact. Fig. 3a shows four thermocouples in different radial positions. Preliminary tests confirmed the assumption of an axisymmetric particle flow. All experiments where dry-ice particles accumulated around the thermocouple were not considered. While validating the experimental setup, tests showed that placing thermocouples in different angular positions mitigated this unwanted effect.

An analysis of the $\mathrm{CO}_{2}$ phase diagram shows that, when the liquid expands from 58.8 bar to the atmospheric pressure of $1 \mathrm{bar}, \mathrm{CO}_{2}$ solidifies through the Joule-Thomson effect and forms dry-ice particles at $-78.5^{\circ} \mathrm{C}$. However, the flow expansion within the nozzle causes an adverse pressure gradient and, thus, at nozzle exit, the pressure is likely to be above the atmospheric value. Considering this, since the spray is a two-phase mixture of solid and gas, 

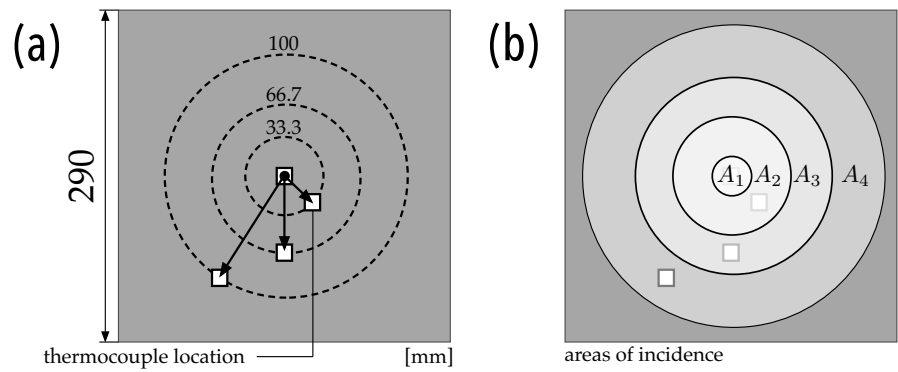

Figure 3. Illustration of thermocouple locations (a) and weighting areas considered in the overall heat transfer analysis.

it would explain why the coolant temperature at nozzle exit stabilizes at a higher value, $T_{c}=-76.8^{\circ} \mathrm{C}$, than $\mathrm{CO}_{2}$ 's sublimation point. This is the value used in the calculations of the heat transfer coefficient.

To investigate the effect of the distance between the spray nozzle and the impinging surface on heat transfer, the values considered are $L=350,400$ and $450 \mathrm{~mm}$.

\section{Measurement Method and Analysis}

Our experiments require a transient heat transfer analysis. The time-frame for applying this cooling technique is up to 6 seconds. This implies calculating the transient $(t)$ heat flux at the impinging surface from local $\left(r_{i}\right)$ temperature measurements for a given impingement distance $(L)$. In Panão and Moreira [4], assuming an adiabatic condition at the bottom surface, the wall heat flux is given by

$$
q^{\prime \prime}\left(t_{n}, r_{i}, L\right)=\frac{2 \beta}{\sqrt{\pi \cdot \delta t}} \sum_{i=1}^{n} \frac{\theta\left(t_{i}, r_{i}, L\right)-\theta\left(t_{i-1}, r_{i}, L\right)}{\sqrt{n-i}+\sqrt{n-i+1}}
$$

where $\beta=\sqrt{\rho k c_{p}}$ corresponds to the thermocouple thermal effusivity, with $\rho, k$ and $c_{p}$ as the density, thermal conductivity and specific heat of the material, respectively. The time interval $\delta t$ depends on temperature data sampling frequency and $\theta\left(t, r_{i}, L\right)=T_{s}\left(t, r_{i}, L\right)-T_{s}\left(0, r_{i}, L\right)$ corresponds to the difference between each instantaneous measurement of the local $\left(r_{i}\right)$ temperature at a given impingement distance $(L)$, and the initial condition at $t=0$. The resultant evolution of surface temperature uses two to three repetitive experiments to ensure reproducibility of the results. The wall heat flux calculated for each temperature profile and, afterwards, used to calculate the spatial-average value for the particle spray impact area,

$$
\left\langle q^{\prime \prime}(t, L)\right\rangle=\frac{1}{A} \sum_{i}\left(q^{\prime \prime}\left(t, r_{i}, L\right) \cdot A_{w}\left(r_{i}\right)\right)
$$

where $A$ corresponds to the total area, $A=\sum_{i} A_{w}\left(r_{i}\right)$, and $A_{w}\left(r_{i}\right)$ is the incidence area associated with each thermocouple, as depicted in Fig. 3b. The positions $r_{i}$ correspond to the thermocouples radial location. An error propagation analysis made for $\left\langle q^{\prime \prime}(t, L)\right\rangle$ resulted in uncertainties starting at $6.5 \%$ and decreasing to a value around $1 \%$ after $100 \mathrm{~ms}$.

Using the temperature measured at the surface $T_{s}\left(t, r_{i}, L\right)$ and the temperature of the two-phase $\mathrm{CO}_{2}$ mixture measured at nozzle exit, $T_{c}$, the spatial-average instantaneous heat convection coefficient $h$ is calculated as

$$
\langle h(t, L)\rangle=\frac{1}{A} \sum_{i}\left(\frac{q^{\prime \prime}\left(t, r_{i}, L\right)}{T_{s}\left(t, r_{i}, L\right)-T_{c}} \cdot A_{w}\left(r_{i}\right)\right)
$$

A final quantity of interest is the total cooling energy removed from the surface,

$$
\left\langle E^{\prime \prime}\right\rangle(\Delta t, L)=\int_{0}^{\Delta t} \frac{1}{A} \sum_{i}\left(q^{\prime \prime}\left(t, r_{i}, L\right) \cdot A_{w}\left(r_{i}\right)\right) \mathrm{dt}
$$

An error propagation analysis made for the spatial average heat transfer coefficient resulted in uncertainties of 2.3-2.7\%, and $0.6-0.8 \%$ for the total energy removed in the cooling process.

\section{Results and discussion}

The first part of the results presented analyzes the surface temperature behavior, and its distribution, to characterize the expected outcome of sublimation spray cooling. Afterwards, the spatial-average evolution of the wall heat flux, heat transfer coefficient and total energy removed in the sublimation process allow characterizing the effect of the impingement distance on the cooling performance. 


\section{Temperature evolution and distribution}

Fig. 4 depicts the average local surface temperature variation for three different impingement distances. The first observation is the significant temperature difference from the plate's center to outer regions in sublimation heat transfer. This is clearer between the axisymmetric center, $r=0 \mathrm{~mm}$ and the first location outwards at $r=33.3 \mathrm{~mm}$ for larger impingement distances, showing the heterogeneous cooling characteristic of impinging jet heat transfer.

Regarding the thermal transient behavior of the surface, $t \approx 1.5 \mathrm{~s}$ appears to set a threshold between a first cooling period with an intense non-linear evolution of surface temperature, and a second period where cooling is less intense, with surface temperature decreasing at a constant and lower rate.

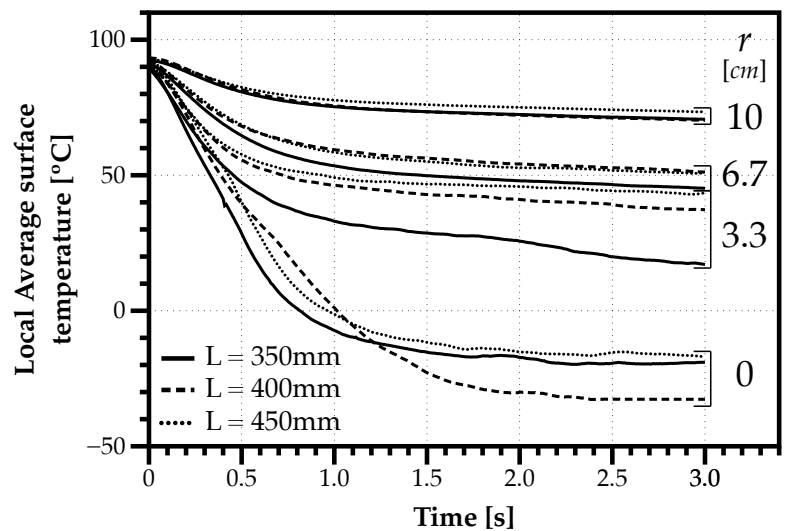

Figure 4. Evolution of local average surface temperature for different impingement distances with an injection duration of $3 \mathrm{~s}$.

Another observation is the range for the surface temperature values $\left(-32^{\circ} \mathrm{C}\right.$ to $\left.-16^{\circ} \mathrm{C}\right)$, which does not reach the sublimation value for $\mathrm{CO}_{2}$ in atmospheric conditions $\left(-78.5^{\circ} \mathrm{C}\right)$. A high-velocity flow of a two-phase (solid-gas) mixture of $\mathrm{CO}_{2}$ impinging on the surface may imply a short residence time of particles in contact with the heated surface for cooling purposes. Compared with cryogen spray cooling, the formation of liquid films allows reaching the saturation temperature (in this case, the boiling temperature, see [5]). However, particles bounce from the surface, justifying the shorter residence time for heat transfer with the surface and, consequently, a higher temperature than the sublimation value.

The experiments performed also considered different durations of injection. Fig. 5 shows the surface temperature decay, $\theta(t)=T_{s}(t)-T_{s}(0)$, for four different durations of injection $(\Delta t=0.5,0.78,1.5,3.75 \mathrm{~s})$ at $r=33.3 \mathrm{~mm}$ and an impingement distance of $400 \mathrm{~mm}$. Since the Start-Of-Injection (SOI), the decay is similar for all pulse durations, and temperature recovery after the end of injection is also similar since it depends on the heat diffusion inside the plate, although the final temperature varies proportionally to the injection duration. Therefore, we assume the wall heat flux evolution for an injection with a longer pulse $\left(\Delta t_{\max }\right)$ can reasonably describe the outcome expected for a shorter pulse, $\Delta t \leq \Delta t_{\max }$. As a fist approximation, this implies considering that the wall heat flux associated with the recovery period has a marginal quantitative effect on the overall outcome. The practical implication is focusing the analysis on the experiments with a longer pulse, assuming that any integration between 0 and $t \leq t_{\max }$ expresses the result expected for the corresponding duration of injection $(\Delta t=t)$ of dry-ice particles onto the heated surface. This is an important consideration in the analysis of the following section on the amount of energy removed from the surface during $\Delta t$, given by Eq. (4).

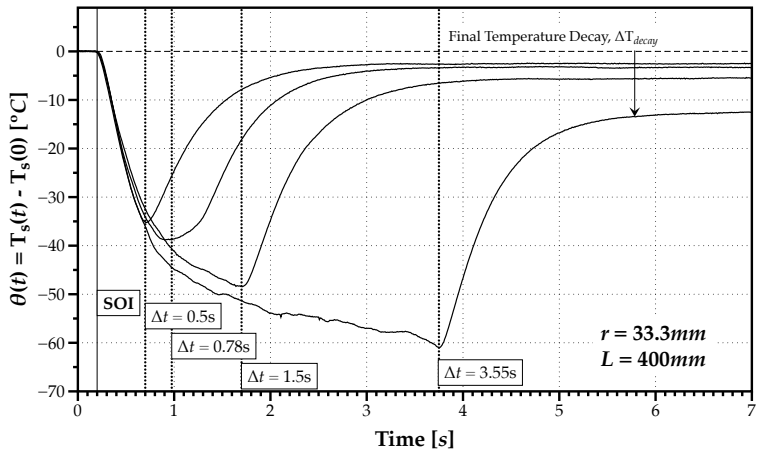

Figure 5. Evolution of the surface temperature decay at $r=33.3 \mathrm{~mm}$ with $L=400 \mathrm{~mm}$ considering different durations of injection $(\Delta t=\{0.5,0.78,1.5,3.75\}[s])$. SOl stands for Start-Of-Injection.

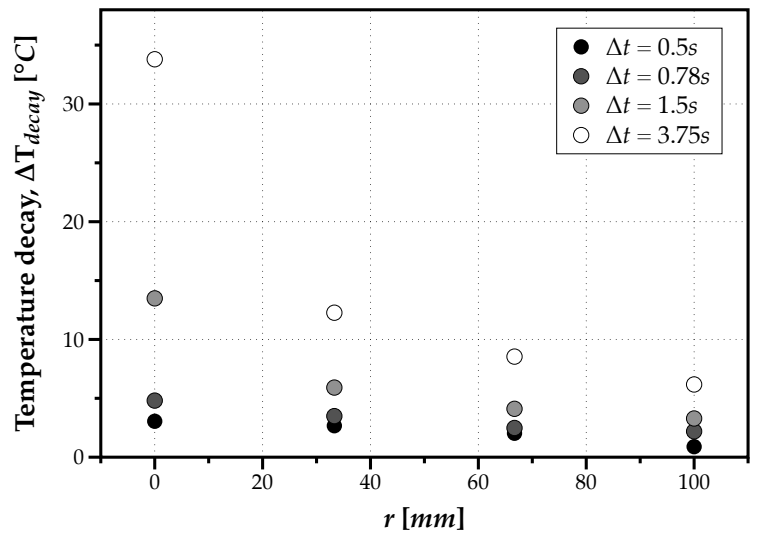

Figure 6. Stabilized final temperature decay after recovery by thermal inertia and within a time window of $6 \mathrm{~s}$.

In Fig. 5, after the end of injection, the final stabilized temperature shows a decay $\left(\Delta T_{\text {decay }}\right)$ produced by the 
impinging dry-ice spray. Fig. 6 depicts the temperature decay in each radial location, considering several durations of injection. A significant growth in temperature heterogeneity within the impact area as the duration of injection increases, particularly in the central region, allows concluding that smaller pulses enable a more uniform cooling in the impingement area, while longer pulses are more adequate for hotspot cooling. The next section focuses on the effect of the impingement distance on sublimation spray cooling heat transfer.

\section{Effect of distance on dry-ice particle spray cooling}

From the surface temperature evolution analyzed in the previous section, Fig. 7a depicts the spatial-average wall heat flux calculated according to Eq. (2). The three curves correspond to different impingement distances and the pattern is similar to the observed in intermittent spray cooling [4], and the magnitude similar to typical values found in cryogen spray cooling [7]. The maximum wall heat flux occurs $0.5-0.6 \mathrm{~s}$ after impact, for all distances $L$, and decreases thereafter, probably due to the $\mathrm{CO}_{2}$ saturation close to the impinging surface, inhibiting sublimation.

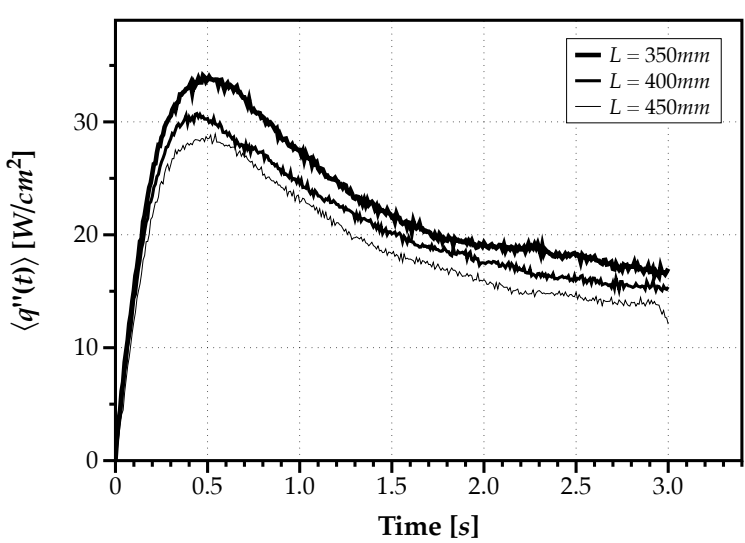

(a) spatial-average wall heat flux

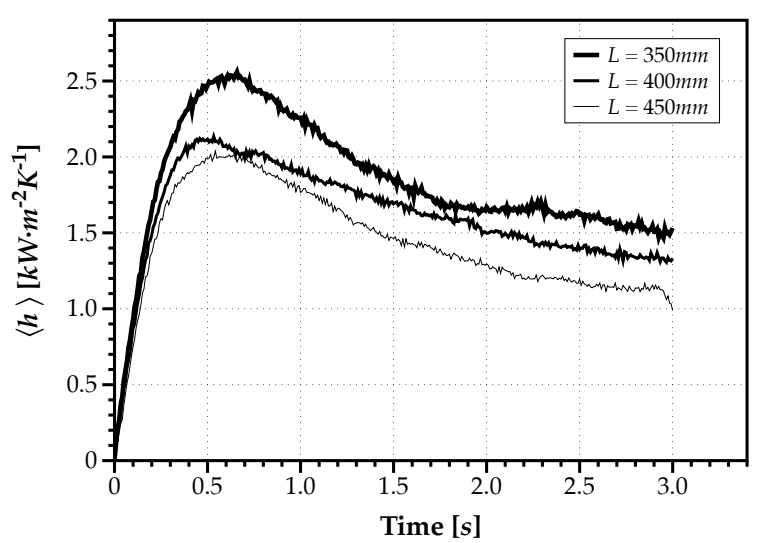

(b) spatial-average heat transfer coefficient

Figure 7. Evolution of the spatial-average heat transfer removed by the dry ice particle spray.

As to the spatial-average heat transfer coefficient, $\langle h\rangle$, Fig. 7b suggests a higher performance for the shorter distance $(L=350 \mathrm{~mm})$, but the behavior is similar for all cases. Moreover, above $t=1 \mathrm{~s}$, $\langle h\rangle$ evolves toward stabilization, suggesting that pulse durations above $3 \mathrm{~s}$ may lead to a steady-state cooling. This is better understood through the analysis of the total energy removed in the cooling process as a function of the duration of injection $(\Delta t)$. Fig. 8 shows a non-linear increase of the energy removed from the surface, and independent of distance, for short pulse durations $\Delta t \leq 0.5 \mathrm{~s}$. Thereafter, the values for $\left\langle E^{\prime \prime}(L, \Delta t)\right\rangle$ increase monotonically with $\Delta t$.

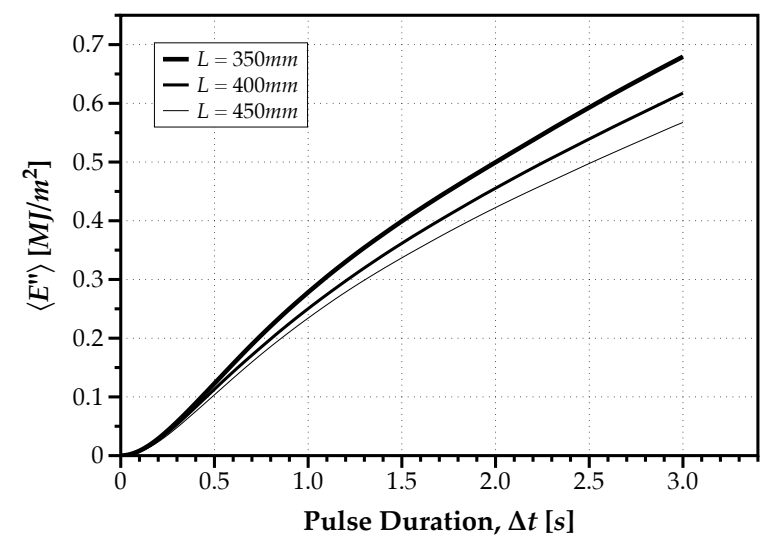

Figure 8. Total cooling energy on the impact of a dry ice spray as a function of the duration of injection.

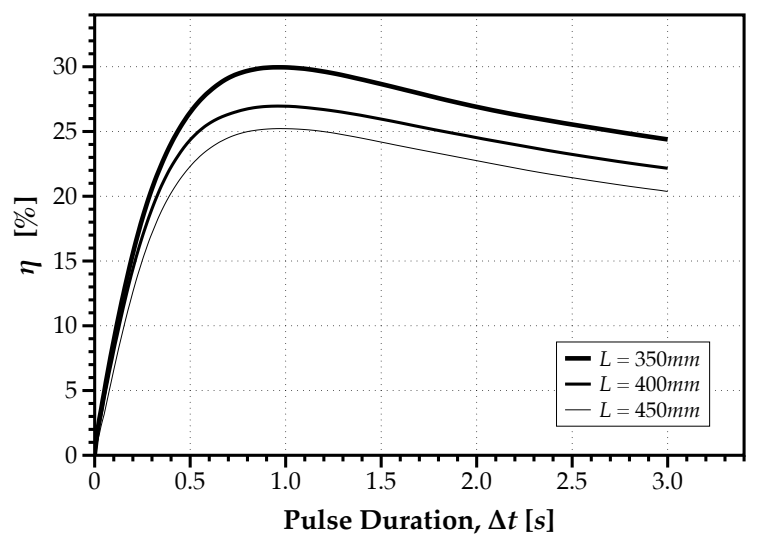

Figure 9. Effect of the impingement distance on the overall cooling efficiency as a function of pulse duration.

Considering the application of this thermal management strategy to mold cooling, the cooling time $(C T)$ in molding is generally proportional to the natural logarithm of a temperature ratio considering the values of injection of material $\left(T_{i n j}\right)$, extraction of molding part $\left(T_{e x t}\right)$, and average mold temperature $\left(T_{m}\right)$,

$$
C T \propto \ln \left(\frac{T_{i n j}-T_{m}}{T_{e x t}-T_{m}}\right)
$$

Thus, if the recurrent use of $\mathrm{CO}_{2}$ would lead to a general decrease of the mold temperature in its cyclic operation, it 
would represent a percentual reduction of the cycle time proportional to the decrease of $T_{m}$ relatively to a reference value without $\mathrm{CO}_{2}$ cooling of the molding cavity surface. This is the research subject of ongoing modeling work. Considering the cooling energy in each injection pulse, and the liquid $\mathrm{CO}_{2}$ quantity expected to form dry ice in order to benefit from sublimation, which is approximately $45 \%$ according to Sherman and Adam [8], the cooling efficiency estimated depends on the pulse duration as

$$
\eta=\frac{\left\langle E^{\prime \prime}(\Delta t, L)\right\rangle}{0.45 \dot{m} h_{s g} \Delta t}
$$

where $\dot{m}$ corresponds to the measured mass flow rate of $\mathrm{CO}_{2}$ and $h_{s g}=573 \mathrm{~kJ} / \mathrm{kg}$ is the sublimation latent heat at atmospheric pressure. The results depicted in Fig. 9 evidence a local maximum around a pulse duration of $\Delta t=1 \mathrm{~s}$. As mentioned before, a longer injection leads to a loss in performance, probably due to the saturation of the mixture flowing environment close to the surface. Although the total cooling energy increases for a longer $\mathrm{CO}_{2}$ injection pulse, Fig. 9 points to a cost in performance. This suggests the possibility of exploring multiple shorter pulses, instead of longer ones, as observed in Panão et al. [9] for cooling with intermittent liquid sprays.

Relatively to the magnitude of cooling efficiency values, compared to other intermittent sprays using liquid [4, 9], the results are similar despite pulse durations in the present experiments are 2-3 orders of magnitude higher. Fig. 9 also points to a performance degradation when injecting the spray farther from the surface. However, this detriment is not significant, since an increase of $30 \%$ of the distance only decreases $\eta$ by $5 \%$.

\section{Conclusions}

In typical thermal management, spray cooling systems often use the injection of liquid onto heated surfaces. However, a liquid film formed after impact mitigates phase-change in most cases and causes losses in cooling performance. Therefore, the present work aims at introducing the application of dry ice particle sprays in the development of thermal management cooling systems. In particular, the work focuses on its transient application within the time frame available for cooling the molding cavity surface in molding processes during its operation time.

The thermal assessment of the technique includes the effect of pulse duration and impingement distance on the temperature distribution, overall heat transfer (in terms of fluxes and heat transfer coefficient), on the total energy removed from the heated surface, and cooling efficiency. Although dry ice sprays are usually associated with cleaning surfaces, the results presented here show their prominent application in transient thermal management cooling systems. Namely,

- Dry ice spray cooling produced different temperature distributions, depending on the duration of injection. With shorter pulses lower than $1 \mathrm{~s}$, the temperature decay is relatively uniform throughout the impact area, while longer pulses produce a more heterogeneous temperature distribution with a higher decay in the central region. This is particularly useful in hotspot cooling.

- The effect of the impingement distance on dry ice spray cooling considers the distances of 350,400 and $450 \mathrm{~mm}$. As expected, a higher impingement distance leads to a higher particle dispersion, leading to a lower thermal performance, thus, lower wall heat flux values. However, it does not affect the thermal behaviour producing a maximum around the instant $0.5 \mathrm{~s}$. This implies an inflection point in the total energy removed by the spray at the injection duration of $0.5 \mathrm{~s}$, distinguishing a correlation for shorter pulses independent on the distance, from another correlation for longer durations depending on the impingement distance parameter.

Given the prominent character of the application of sublimation in thermal management systems, future and ongoing research work includes: the thermal modeling of introducing this additional cooling in molding processes to reduce the cycle time; a more detailed characterization of the dry ice particle spray, with particular emphasis on the impact region; and the effect of the diffusor geometry on the dynamics of dry ice particle and dispersion patterns.

\section{Acknowledgements}

The authors would like to acknowledge to the financial support of project EMCool - Efficient Mold Cooling (POCI01-0247-FEDER-011375).

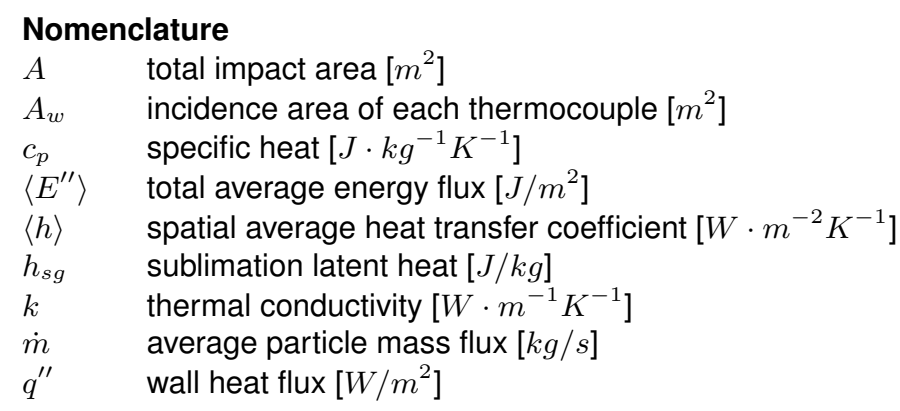




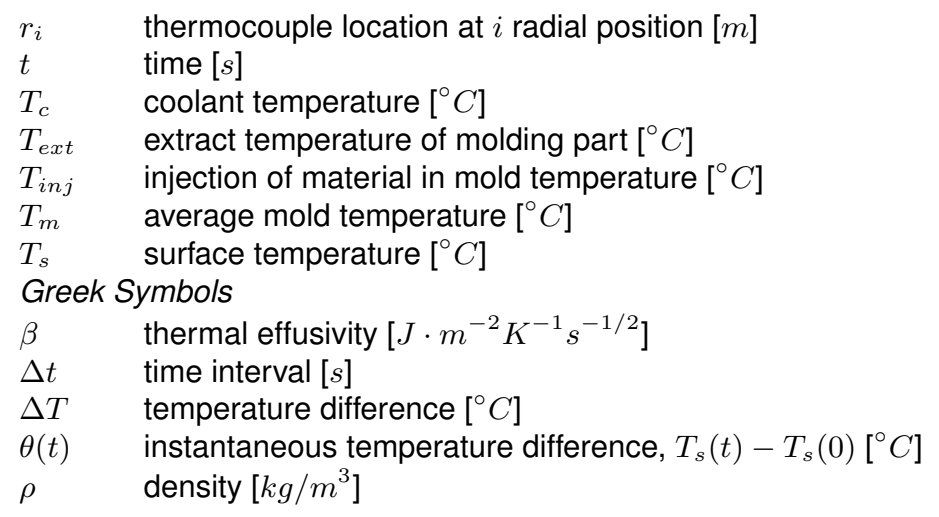

\section{References}

[1] Stanley, J. R., Praller, A., 2015, Spot cooling with $\mathrm{CO}_{2}$, Plastics Engineering, July/August, pp. 20-22.

[2] Sherman, R., Grob, J., Whitlock, W., 1991, Dry surface cleaning using $\mathrm{CO}_{2}$ snow, J. Vac. Sci. Technol. B 9, pp. 1970-1977.

[3] Kim, D., Lee, J., Experimental investigation of $\mathrm{CO}_{2}$ dry-ice assisted jet impingement cooling, Applied Thermal Engineering 107, pp. 927-935.

[4] Panão, M. R. O., Moreira, A. L. N., 2009, Intermittent spray cooling: A new technology for controlling surface temperature, International Journal of Heat and Fluid Flow 30 (1), pp. $117-130$.

[5] Aguilar, G., Wang, G.-X., Nelson, J. S., 2003, Dynamic behavior of cryogen spray cooling: Effects of spurt duration and spray distance, Lasers in Surgery and Medicine 32 (2), pp. 152-159.

[6] Majaron, B., Svaasand, L. O., Aguilar, G., Nelson, J. S., 2002, Intermittent cryogen spray cooling for optimal heat extraction during dermatologic laser treatment, Physics in Medicine and Biology 47 (18), pp. 3275-3288.

[7] Zhou, Z.-F., Wang, R., Chen, B., Yang, T., Wang, G.-X., 2016, Heat transfer characteristics during pulsed spray cooling with R404A at different spray distances and back pressures, Applied Thermal Engineering 102 (C), pp. 813-821.

[8] Sherman, R., Adam, P., Carbon Dioxide Snow Cleaning - The next Generation of Clean, Precision Cleaning '95 Proceedings, pp. 271-300.

[9] Panão, M. R. O., Correia, A. M., Moreira, A. L. N., 2012, High-power electronics thermal management with intermittent multijet sprays, Applied Thermal Engineering 37, pp. 293-301. 